\section{$\underset{\substack{\text { hommes } \\ \text { \& migrations }}}{ }$}

\section{Hommes \& migrations}

Revue française de référence sur les dynamiques

migratoires

$1312 \mid 2015$

Diasporas iraniennes

\title{
Ghazel : l'artiste mobile, l'art de la mobilité
}

\section{Amin Moghadam}

\section{(2) OpenEdition \\ Journals}

\section{Édition électronique}

URL : http://journals.openedition.org/hommesmigrations/3500

DOI : 10.4000/hommesmigrations.3500

ISSN : 2262-3353

\section{Éditeur}

Musée national de l'histoire de l'immigration

\section{Édition imprimée}

Date de publication : 1 octobre 2015

Pagination : 91-97

ISBN : 978-2-919040-33-9

ISSN : $1142-852 X$

\section{Référence électronique}

Amin Moghadam, «Ghazel : l'artiste mobile, l'art de la mobilité », Hommes \& migrations [En ligne], 1312 | 2015, mis en ligne le 01 octobre 2018, consulté le 02 mai 2019. URL : http://

journals.openedition.org/hommesmigrations/3500; DOI : 10.4000/hommesmigrations.3500 


\section{GHAZEL \\ L'ARTISTE MOBILE, L'ART DE LA MOBILITÉ}

par AMIN MOGHADAM, géographe à l'Unité de recherche migrations et société (URMIS), Aix-Marseille Université.

La création artistique contemporaine se nourrit des circulations des artistes entre plusieurs territoires, à l'image du travail de Ghazel, artiste visuelle iranienne. Entre I'Iran, la France et Dubaï, portrait d'une artiste qui se joue des espaces et des identités, faisant de sa propre mobilité le motif de ses œuvres.

Depuis l'avènement de la République islamique en 1979, malgré les difficultés de déplacement en dehors des frontières iraniennes, les artistes visuels iraniens voyagent souvent dans les grandes métropoles du monde, rencontrent d'autres expressions artistiques et s'en inspirent. Pour l'artiste, ce phénomène va de pair avec la diversification des territoires de sa pratique artistique, depuis son lieu de vie jusqu'à la production, la commercialisation et la reconnaissance publique de son œuvre. Ainsi la mobilité professionnelle devient tributaire des mobilités géographiques qui sont nécessaires à sa réussite ${ }^{1}$. L'étude des trajectoires individuelles des artistes contemporains du Moyen-Orient permet de comprendre comment cette complexité s'ins- crit dans leur parcours artistique et esthétique et de "mesurer comment les parcours géographiques se calquent sur les mobilités professionnelles ascendantes ou descendantes des artistes".

De nombreux artistes iraniens ont décidé de vivre dans les villes occidentales tout en gardant des liens réguliers avec leur pays d'origine, l'Iran. L'expérience du voyage, de la rencontre et, en quelque sorte, du cosmopolitisme leur permet de jongler entre leurs acquis intellectuels à l'extérieur de leur pays et ceux de leur culture d'origine ancrée dans une histoire nationale particulière. Leur géographie mentale est donc multicontextualisée et leur œuvre multisituée ${ }^{3}$. Ainsi, leur déplacement et leur œuvre créent du sens, ou bien multiplient les 
significations investies dans l'œuvre. Par ailleurs, la multiplication des déplacements et des voyages devient aussi une problématique à laquelle certains artistes accordent une attention particulière comme dans l'œuvre de Ghazel dont je vais tenter dans cet article de décrire quelques aspects.

Ghazel est tout d'abord une chère amie qui m'a permis de suivre l'évolution de son travail depuis plusieurs années. Cette analyse repose sur de nombreuses discussions informelles entre nous mais aussi sur quelques entretiens davantage encadrés qu'elle m’a accordés à Dubaï, Téhéran et Paris entre 2011 et 20144. L'œuvre de Ghazel m'intéresse pour trois raisons : d'abord son vécu et ses ressentis personnels et la manière dont elle les interprète et les représente dans son travail ; ensuite, la question de l'appartenance nationale et des mobilités et le regard qu'elle porte sur elles à travers son œuvre ; enfin, la réception de ses œuvres par un public de plus en plus large à travers le monde, dont les codes et les sensibilités culturels peuvent varier d'un pays à l'autre.

\section{Un voyage qui commence en Iran}

Ghazel est une artiste qui réside entre Paris et Téhéran et dont les œuvres sont présentées dans plusieurs galeries et institutions à travers le monde, notamment au sein de la collection permanente du Musée national de l'histoire de l'immigration à Paris. Elle est arrivée en France en 1986 pour faire des études et s'y est installée. Ghazel 
voyage aujourd'hui régulièrement dans plusieurs pays, en particulier à Dubaï, à Téhéran et à Paris pour des raisons personnelles ou professionnelles. Ses mobilités font partie de son œuvre : elle interroge, à travers l'usage des œuvres visuelles comme des cartes géographiques, des vidéos ou des performances, une vision essentialiste de la nation et de l'appartenance nationale qu'elle confronte avec sa volonté d'être une "citoyenne du monde".

Née dans une famille aisée de Téhéran, Ghazel est allée dans une école internationale en primaire et au collège où l'enseignement était dispensé en anglais. Au lendemain de la révolution de 1979, toutes les écoles étrangères sont fermées et Ghazel doit terminer ses études secondaires dans un lycée public avant d'être admise à l'université Al-Zahra de Téhéran au sein du département de design. Ghazel a souligné que, venant d'une famille aisée du nord de Téhéran, elle s'est sentie étrangère dans cette université iranienne où elle a rencontré des gens qui venaient de toutes les villes d'Iran, des villes dont elle n'avait jamais entendu parler" : “J'ai même remarqué que j'avais un léger accent quand je parlais dans ma langue maternelle. Je me suis rendu compte que j'avait vécu pendant dix-neuf ans dans une bulle protégée dont il était difficile de s'échapper ${ }^{6}$."

Très vite, elle se sent "aliénée" selon ses propres mots, et décide de partir pour l'Europe où elle entame des études à l'université Paul-Valéry de Montpellier. Elle obtient son diplôme national supérieur d'expression plastique (DNSEP) à l'École des Beaux-Arts de Nîmes en 1992 et plus tard une licence en études cinématographiques.

\section{Me : la mise en scène de soi et de l'autre.}

C'est pourtant avec la distance d'une étrangère en France que Ghazel commence à s'intéresser aux problématiques de sa société d'origine. À titre d'exemple, dans une série de courtes vidéos inti- tulée $M e$, elle met en scène une figure muette, toujours la même, qui vient jouer une scène de la vie quotidienne que l'artiste a connue elle-même en Iran. Ces vidéos naissent des paradoxes de la vie quotidienne de Ghazel, entre son travail d'artthérapeute dans un centre de réinsertion pour les jeunes délinquants à Téhéran et sa vie de jeune fille iranienne de la bonne société iranienne.

À travers Me se dessine avant tout le portrait d'une artiste, femme et Iranienne, avec des séquences de vie tournées dans des lieux qui lui sont familiers. Mais, au fur et à mesure que ces vidéos se multiplient, elles traitent de plus en plus de la vie quotidienne des femmes iraniennes toujours représentées par Ghazel, elle-même couverte par le tchador qu'elle considère comme une couleur locale iranienne et non pas nécessairement comme un signe religieux imposé aux femmes iraniennes. Faits et gestes du quotidien tissent la trame d'une existence qui fait malgré tout la part belle au plaisir, à l'insolite (ski nautique en tchador), mais aussi la rumeur du monde et de l'histoire (une femme armée arpentant péniblement une piste de ski avec héroïsme), toujours traitée par Ghazel avec humour.

Me ne prétend pas représenter la femme iranienne, mais nous force à regarder autrement ces scènes quotidiennes : images stéréotypées de la femme iranienne, images "Les déplacements, c'est ma vie, ça fait vingt-cinq ans que j'ai quitté l'Iran, mais j'ai toujours un pied en Iran, je suis ça, je ne peux pas le dissocier de mon travail. Un pied ici et un pied là-bas. Je déteste quand on me présente comme une 'artiste française d'origine iranienne', je suis iranienne et j'ai un passeport français, c'est tout! de l'intimité que surprend le spectateur, prégnance du tchador comme signe vestimentaire incontournable. "Life is fun!" s'écrie l'artiste en tchador montée sur un cheval de manège. Néanmoins, c'est avec cet "uniforme", comme l'appelle Ghazel, que la femme iranienne fait son entrée dans l'espace public depuis la révolution de 1979. 


\section{Des loyautés multiples dans I'œuvre de Ghazel}

"Les déplacements, c'est ma vie, ça fait vingtcinq ans que j'ai quitté l'Iran, mais j'ai toujours un pied en Iran, je suis ça, je ne peux pas le dissocier de mon travail. La nationalité, on ne peut pas la remplacer, une autre nationalité peut se rajouter mais c'est tout! Tu deviens un être hybride, tu deviens les deux ou plusieurs à la fois, mais on n'efface pas l'une pour accepter une autre. Aux États-Unis, il y a des gens qui changent leur nom et qui veulent devenir américains. Moi, je suis étrangère partout et cela s'entend déjà à travers mon accent dans les différentes langues que je parle", m'explique Ghazel dans un entretien. Son hybridité et son sentiment d'être "la citoyenne du monde" ne s'expriment pourtant pas au détriment de ses sentiments patrio-

La cosmopolitisation du tiques envers l'Iran. Après parcours de Ghazel combine vingt-trois ans de vie en France,

lescultures nationales Ghazel obtient la nationaet locales, la langue de son lité française pour des raisons

pays d'origine, son envie pratiques tout en conservant de retourner dans sa maison sa nationalité iranienne : "Je au bord de la mer caspienne.

Cette prise de conscience des identités multiples

dont elle dispose, Ghazel la met au service de son art, de son travail. pense qu'il y a eu un changement dans mon travail avec l'obtention de la nationalité, parce que tout ce questionnement [à propos de son travail] est arrivé aussi avec des phrases comme 'Ah, vous êtes française et d'origine iranienne?', et je disais: 'Mais non, je suis iranienne, mais jai un passeport français', et bien sûr la culture française est en moi après vingt-cinq ans. Mais tu ne sais pas comment te présenter aux gens, je ne vais pas faire une fête parce que je suis devenue française! Ce nétait pas un grand événement pour moi, je l'ai vécu d'abord comme une trahison envers mon père et mes racines, et c'est pour ça
Ski, Me (1997-2000).

(c) ADAGP, 2016.

que j'ai commencé à travailler sur les racines. Mon frère a toujours refusé de se faire naturaliser, et moi j'ai refusé pendant longtemps, j'avais une carte de séjour."

$\mathrm{Si}$, à travers la mise en représentation des possibilités d'identification légale, Ghazel porte un regard critique sur les loyautés restreintes que les Étatsnations réclament de leurs citoyens, dans son propre récit de vie, elle n'a pas caché son enthousiasme quand l'obtention de la nationalité française lui a permis de voter en France. Elle se souvient d'avoir pleuré de tristesse lors de l'élection de Nicolas Sarkozy, alors qu'elle n'avait pas pu voter en tant qu'étrangère. Mais la recherche de ce qu'elle appelle son "home" est relancée dès lors qu'elle ressent une situation en tant quétrangère, ce qui la conduit à repartir parfois vers ses lieux d'origine: "Quand j'ai fait la demande de la nationalité française, ça faisait vingt ans que j'habitais en France et je commençais à 
avoir envie de voter, je me disais: 'Tu dois accepter que tu n'es pas toujours une nomade', et cela a coïncidé avec un contrat que jai signé pour un appartement à Paris et pour la première fois à 40 ans, au bout de vingt ans de vie en France, j'ai acheté un matelas, j’ai acheté un frigo, j'avais toujours vécu dans les appartements meublés et des résidences d'artiste. Donc, j’ai créé des racines et, en quelque sorte, en achetant des meubles. En 2009, j'ai eu ma nationalité française peu de temps après avoir commencé cette série de travail. Je suis retournée en Iran, au bord de la Caspienne, là où c'est vraiment ma maison, avec un grand $M, H O M E$, c'est là où je me sens protégée, et j'ai commencé à faire du grand format de dessins que je n'avais pas fait depuis longtemps."

La cosmopolitisation ${ }^{7}$ du parcours de Ghazel combine les cultures nationales et locales, la langue de son pays d'origine, son envie de retourner dans sa maison au bord de la mer Caspienne. Cette prise de conscience des identités multiples dont elle dispose, Ghazel la met au service de son art, de son travail. L'artiste s'apparente donc à une "citoyenne flexible", dont les possibilités d'identifications multiples se reflètent dans son discours mais aussi dans ses créations, comme le résultat d'une série d'intentionnalités, aussi bien pour exprimer ses envies et ses besoins personnels que pour être comprise par son public. Cette "optique cosmopolitique" de Ghazel, pour reprendre l'expression d'Ulrich Beck ${ }^{9}$, contribue à une certaine mâ̂trise du monde tout en étant construite à partir de bases identitaires élémentaires telles que la famille, la classe sociale et la nation.

\section{Mobilité, une matière pour l'art}

Les difficultés que Ghazel rencontre pour obtenir un titre de séjour en France, pendant les voyages qu'elle doit effectuer avec un passeport iranien, ont donné naissance à tout un ensemble de représentations artistiques qui interrogent la question des frontières, des signes nationaux et des repré- sentations cartographiques des pays. Ces thématiques se renforcent dans son parcours à la suite de sa rencontre avec les réfugiés et les sans-papiers afghans à Paris, qui a abouti aux performances mettant en récit la vie de ces derniers.

Dans l'un des premiers travaux qui aborde ces thématiques, Ghazel réalise en 1997 une série d'affiches intitulée "Urgent", aujourd'hui exposée dans la collection permanente du Musée national de l'histoire de l'immigration à Paris, qui illustrent avec humour et dérision les difficultés qu'elle a rencontrées en France pour obtenir un titre de séjour. Sous la forme d'une série d'affiches, Ghazel se lance dans une création autour de la dernière solution qui lui reste pour pouvoir régulariser sa situation : le mariage blanc. Après la réception de l'avis d'expulsion, Ghazel décide de mettre des annonces pour un mariage blanc et réalise sa première affiche. L'artiste poursuit son projet, déclinant les affiches en plusieurs versions jusqu'en 2002, date à laquelle elle reçoit une carte de résidence pour dix ans. Elle renverse alors les données et propose à son tour un mariage à un clandestin afin qu'il puisse obtenir une carte de séjour.

"Procédant par détournement, souligne Isabelle Renard, elle réutilise dans cette oeuvre les acronymes, typiques de la langue française, et convertit avec humour ces sigles passés dans le langage courant: le SDF se transforme en SPF, 'sans-papiers fixe', allusion au fait qu'elle a eu droit à des papiers provisoires en tant qu'artiste. RDD signifie dans le langage de l'artiste une 'résidente à durée déterminée'. Selon les affiches et leur évolution dans le temps, celles-ci insistent sur le type d'homme recherché (avec comme seule exigence qu'il ait des papiers), ou au contraire la dévoilent elle (son image apparait, partiellement ou entièrement, son origine moyen-orientale est mentionnée, puis le fait qu'elle soit artiste $)^{10}$."

Au fur et à mesure qu'elle développe sa pensée autour des questions d'enracinement, d'appartenance et de déplacement, elle prend de la distance par rapport à ses propres expériences de vie 
et adopte des formes d'expression artistique qui évoquent des expériences communes de voyage et de déplacement à l'échelle de la planète. Dans une série nommée La géopolitique des racines-no man's land (2011), elle remet directement en cause les frontières établies par les États sur une carte géographique du monde en les représentant par les drapeaux nationaux. En ajoutant sur ces cartes des éléments sous forme de dessins qui illustrent les appartenances et les liens affectifs multiples possibles entre plusieurs territoires, Ghazel montre le décalage qui existe entre la conception que les États ont des territoires nationaux et leur vision de l'allégeance et la réalité du vécu des individus qui les traversent.

Dans une autre série d'imprimés sur des T-shirts appelée Snap Shots (2010), elle observe avec ironie les procédures de naturalisation qu'elle a dû affronter personnellement en France avant d'être naturalisée, mais aussi l'impasse de ces procédures pour d'autres catégories d'immigrés en difficulté. À travers ces imprimés sur les T-shirts, elle interroge la nationalité légale sous forme d'un lien contractuel rendu possible, impossible ou en attente pour ces personnages variés qui portent ces T-shirts.

Ghazel a réalisé en 2012 des performances appelées Road Movie au Musée national de l'histoire de l'immigration pendant lesquelles elle racontait les récits de vie des Afghans et des Iraniens qui ont traversé plusieurs pays par les voies illégales avant d'atteindre les pays européens du Nord. Elle raconte comment la langue persane avait établi le lien avec les immigrés qu'elle a rencontrés: "Quand dans Road Movie j'ai travaillé avec des immigrés afghans, je voulais travailler avec eux aussi parce que nous partageons une langue, et j'avais envie de travailler dans ma langue maternelle. Quand j'ai demandé à une amie qui travaillait dans le centre des enfants immigrés de me présenter des gens, il y avait des Bangladeshis et des Pakistanais qui me disaient: 'Mais pourquoi pas nous?' Je leur ai répondu: 'Le choix a

Road Movie IV, performance Iros de La Nuit des Musées, Palais de la Porte Dorée, 2012. () Awatef Chengal.

été fait en raison de ma langue maternelle que je partage avec les Afghans.' C'était important pour moi. En 2010, cétait la première fois que je travaillais dans ma langue maternelle depuis le début de mes études aux Beaux-Arts. J'avais tout d'un coup besoin de montrer que cette 'Française d'origine iranienne' est iranienne! J'avais besoin de cette revendication."

Dans ces dernières séries, le déplacement n'est plus une manière de prendre de la distance pour aborder les questions qui la préoccupent dans sa société d'origine, c'est le déplacement même et ses modalités qui deviennent une matière pour l'art.

\section{Les difficultés d'une œuvre qui circule : I'universalité en question}

Au-delà du ressenti personnel, Ghazel se pose la question de la réception de son œuvre par un public de plus en plus large qu'elle rencontre lors de ses voyages et de ses expositions dans différentes villes du monde. Une angoisse se fait ressentir à l'idée de trouver un compromis entre un niveau d'intelligibi- 
lité globale (intelligible pour une sphère globale) et un enfermement dans un particularisme irano-centré qui, même s'il est bien reçu, compte tenu souvent de sa dimension exotique et essentialisée par les spectateurs non-iraniens, prive l'artiste de son besoin d'exprimer la complexité de son travail.

À cet égard, Ghazel explique l'évolution de sa propre esthétique: “Aujourd”hui, je trouve que l'esthétique de mon travail est plus universelle qu'avant, il y a l'arbre, le soleil. Il y avait une Indienne tout à l'heure [dans une galerie à Dubaï] qui me disait que ces cartes sont l'histoire de sa vie. Quand jétais aux Beaux-Arts, je nétais pas comprise, on ne comprenait pas mes tulipes noires par rapport aux tulipes rouges ${ }^{11}$. J'ai dû simplifier mon langage. Je l'avais déjà fait avec la série Me mais avec une couleur locale qui était le tchador. Maintenant j'ai appris à moins jouer avec mes identités. Avant, dans mes vidéos de la série Me, je parlais plus de moi, je faisais mon portrait mais dans ces nouveaux dessins, ce n'est plus tellement moi et c'est moins personnel. Cette carte aurait pu être en arabe ou en chinois et non pas en persan." L'artiste est ainsi amené à réfléchir en amont, dès la création, à la réception différenciée lorsque son œuvre circulera dans ces espaces globalisés. Comment être iranien sans l'être est la question à laquelle l'artiste iranien d'aujourd'hui tente de répondre. Comment devenir un artiste universel en partant des particularismes locaux ? Comment rendre intelligible cette articulation des géographies d'inspiration pour le spectateur "globalisé" dont la sensibilité culturelle et artistique n’a pas par définition la même profondeur que celle de l'artiste quant à son rapport à sa propre culture ? Ce sont des questions auxquelles l'artiste iranien est amené à répondre et qu'il semble intégrer en amont de son travail. Certains usent de la référence aux origines, d'autres brouillent les traces qui conduiraient à une interprétation univoque de leur œuvre, comme c'est bien le cas ici dans l'œuvre de Ghazel. 